\title{
Correction to: A review of interfacial bonding mechanism of bamboo fiber reinforced polymer composites
}

\author{
Lijuan Liu $\mathbb{D} \cdot$ Zhiqing Yuan $\cdot$ Xiaokun Fan $\cdot$ Chaoquan Pan $\cdot$ Xinyi Li
}

Published online: 27 November 2021

(C) Springer Nature B.V. 2021

\section{Correction to:}

Cellulose

https://doi.org/10.1007/s10570-021-04242-6

In the original publication, the captions of Figs. 3, 4, 5, $6,8,10$ and 11 were processed incorrectly. The correct captions with the figures are provided in this correction. 


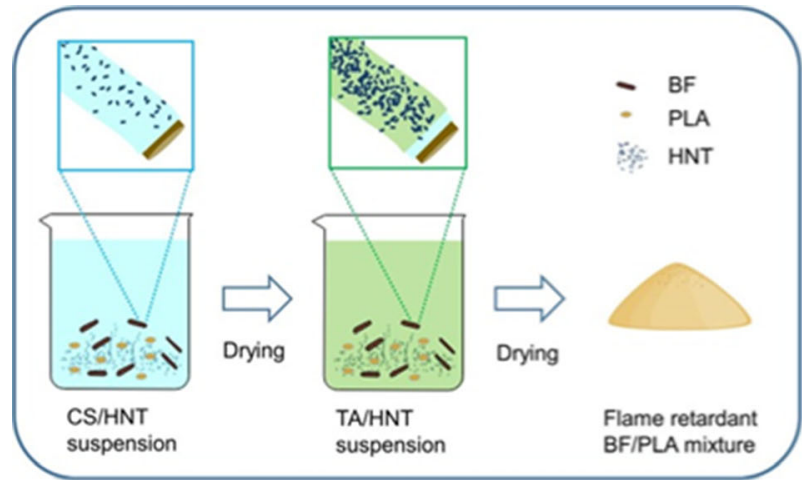

(a)

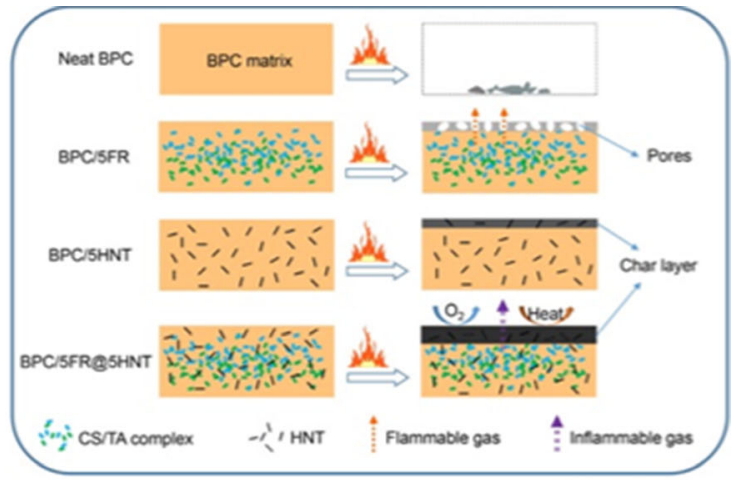

(b)

Fig. 3 a Preparation of flame retardant. b Diagrammatic sketch of combustion mechanism about composites. Figure 3 reproduced with permission from John Wiley and Sons. (Jin et al. 2020)

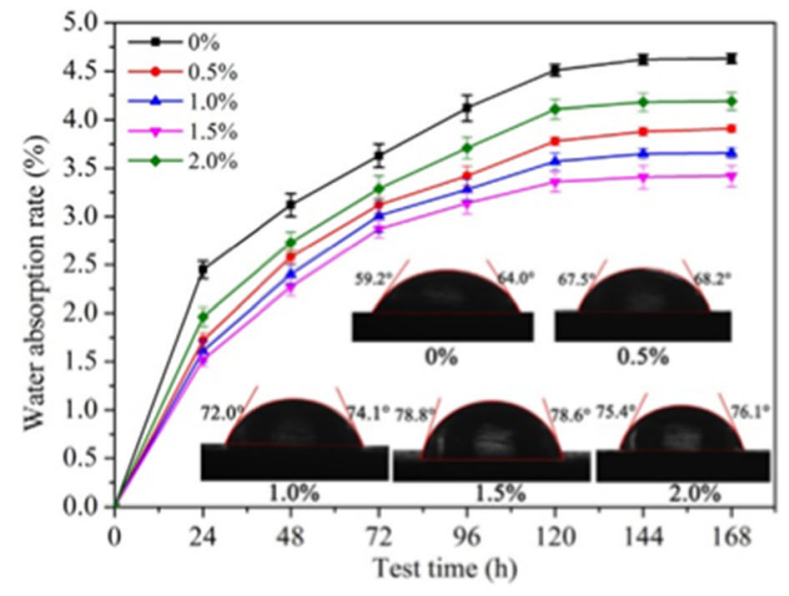

(a)

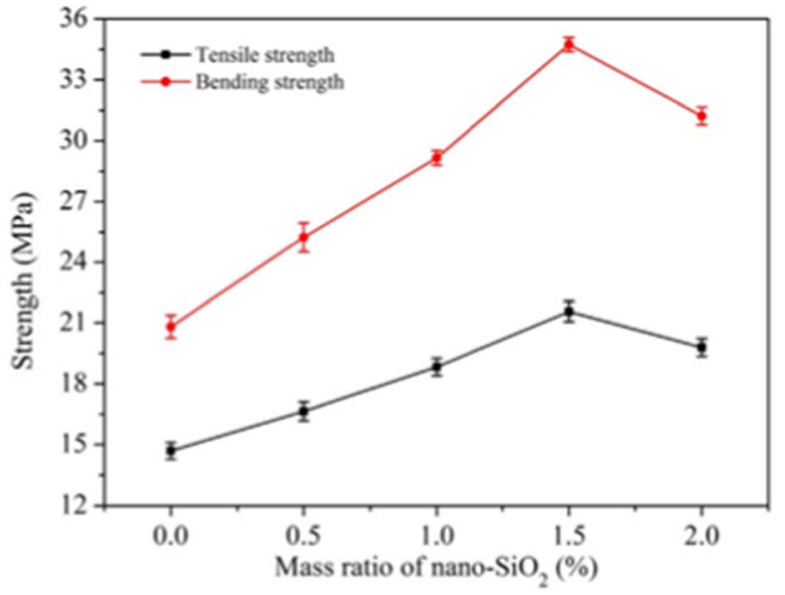

(b)

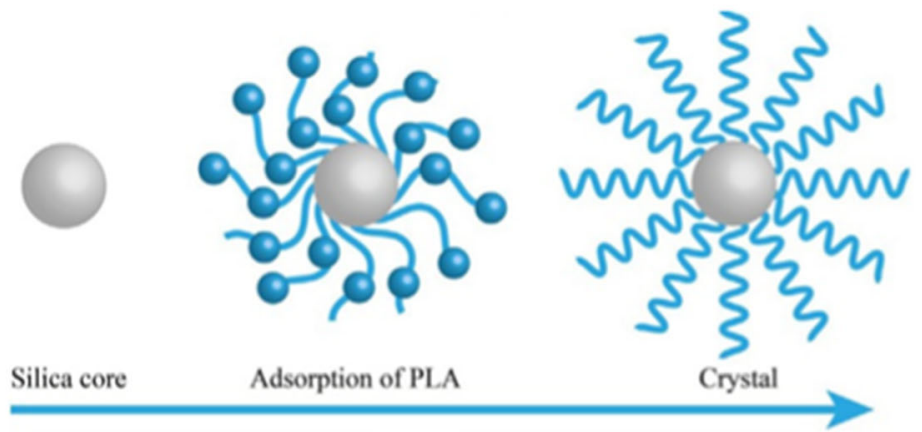

(c)

Fig. 4 a Influence of various nano silica contents on contact angle and water-absorption quality of composites. b Influence of various nano silica contents on mechanical properties of composites. c Crystallization process of polylactic acid. Figure 4 reproduced with permission from Elsevier. (Zuo et al. 2020) 


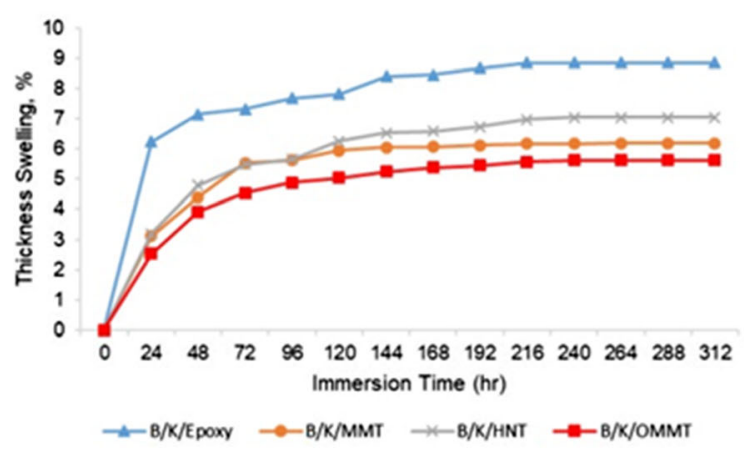

(a)

Fig. 5 a Thickness swelling of various composites. b The storage modulus of composite formed by 4 layers of woven glass fiber (GGGG), 2 layers of woven glass fiber and 2 layers of garded ensete web (GGEE), and glass/ ensete /ensete/glass

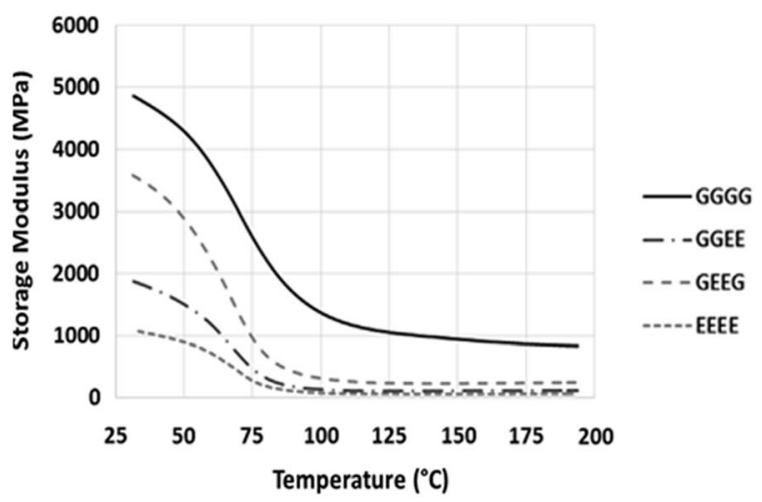

(b)

multi-layer (GEEG) and 4 layers of garded ensete web (EEEE) reinforced unsaturated polyester hybrid composites. Figure 5 reproduced with permission from Elsevier (Chee et al. 2020; Tolera et al. 2021)

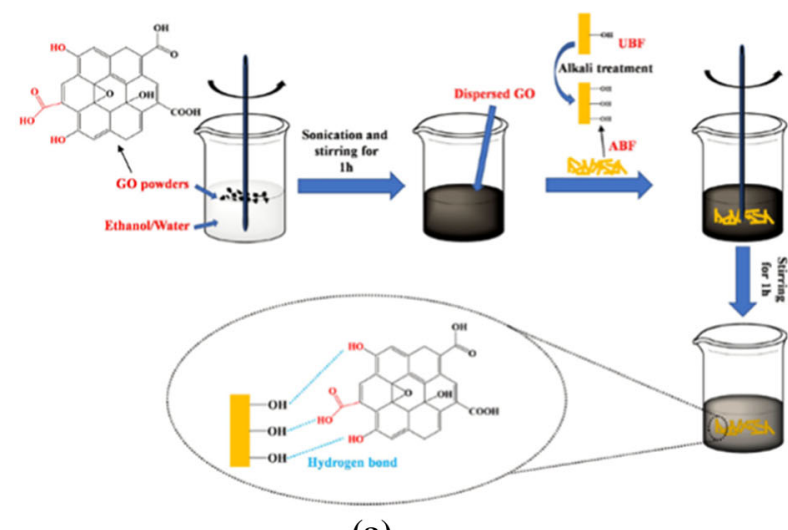

(a)
Fig. 6 a Diagrammatic sketch of the preparation about the graphene oxide (GO) grafted onto alkali-treated $\mathrm{BF}(\mathrm{GO}-\mathrm{ABF})$. SEM images of b, c untreated BF, d, e 0.05, 0.1 GO-ABF. f, $\mathbf{g}$, tensile, flexural tests of various composites. $0.05,0.1$ and 0.3
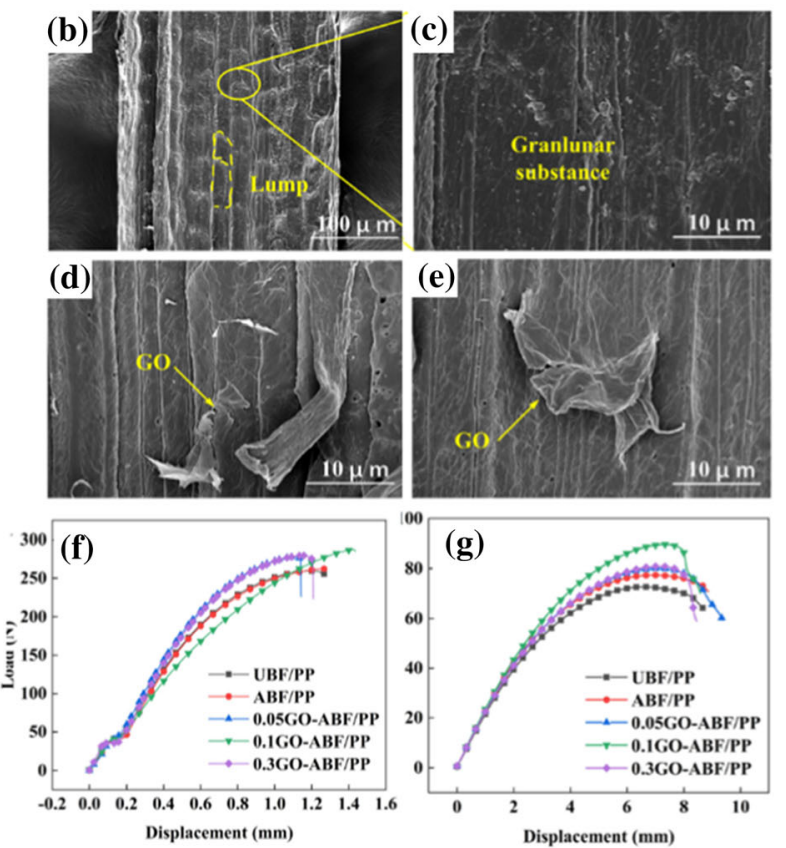

GO-ABF based on the GO concentrations of $0.05,0.1$ and 0.3 wt. $\%$ respectively. Figure 6 reproduced with permission from John Wiley and Sons. (Wang et al. 2020) 


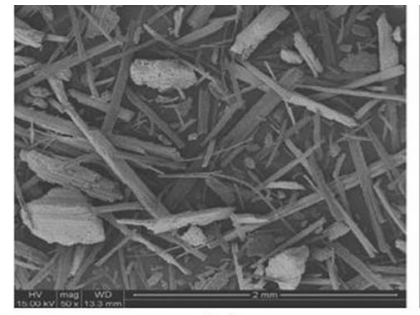

(a) $\times 50$

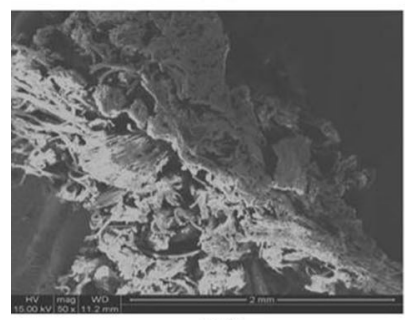

(c) $\times 50$

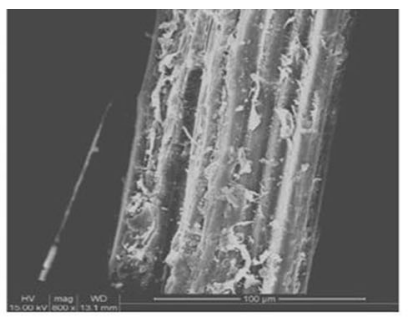

(b) $\times 800$

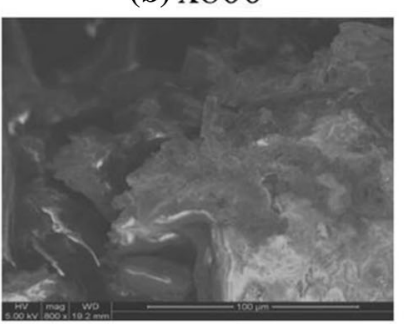

(d) $\times 800$
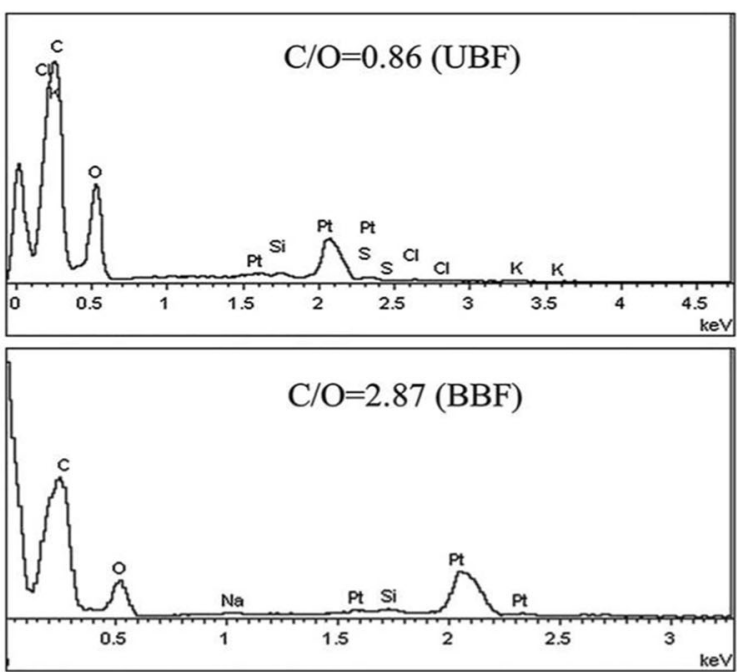

(e)

Fig. 8 SEM micrographs: a, b, untreated bamboo flour $(\times 50)$, $(\times 800), \mathbf{c}, \mathbf{d}$ benzylated bamboo flour $(\times 50),(\times 800)$. e Energy dispersive $X$-ray spectra of unmodified bamboo flour (UBF) and

benzylated bamboo flour (BBF) (Sun et al. 2016). Figure 8 reproduced with permission from John Wiley and Sons
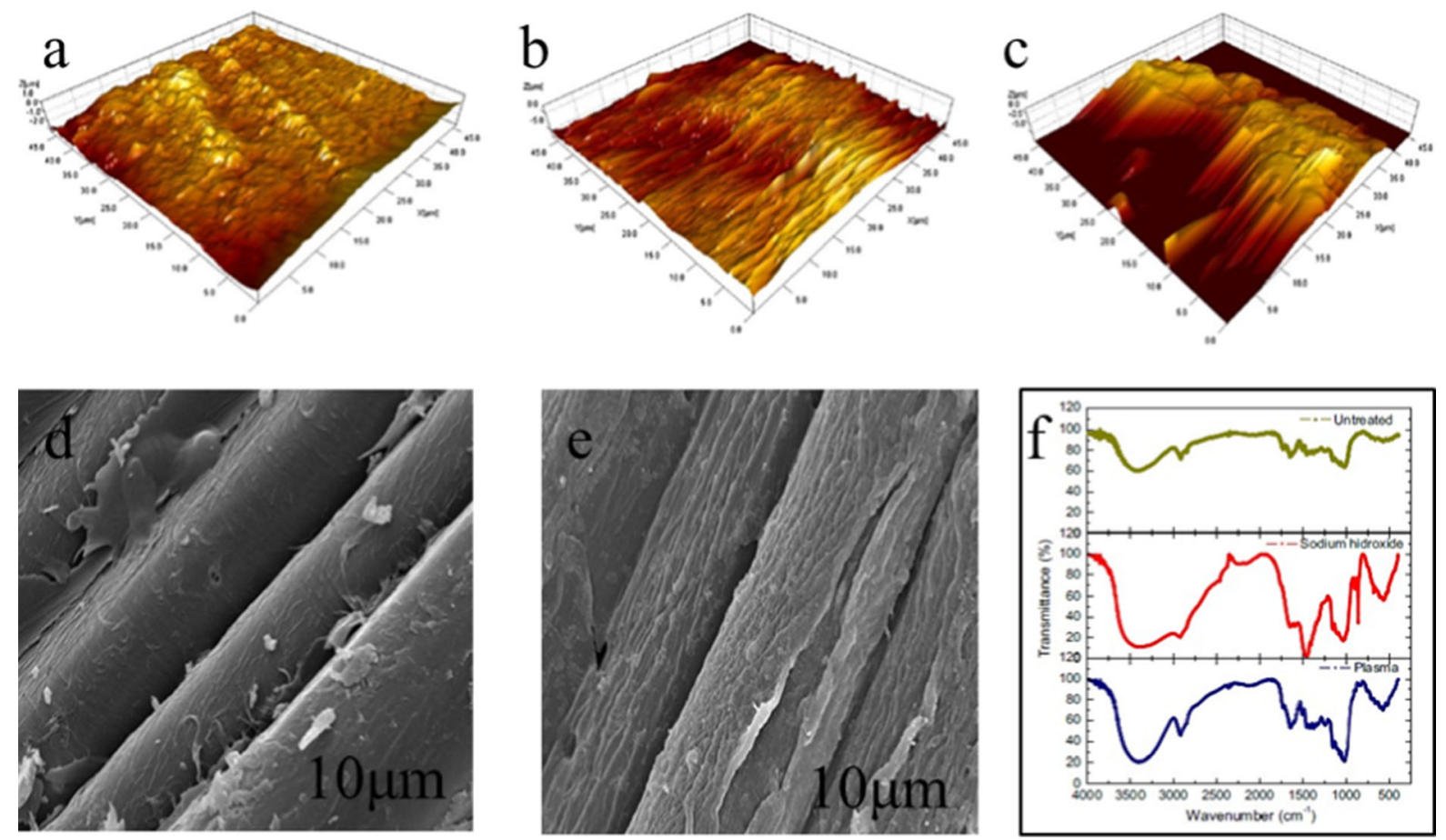

Fig. 10 a, b, c Atomic force microscopy images of untreated treatment, alkaline treatment, plasma treatment of BF. d, e SEM absorption bands. Figure 10 reproduced with permission from Elsevier. (Sa'nchez et al. 2019; Suwan et al. 2020) 


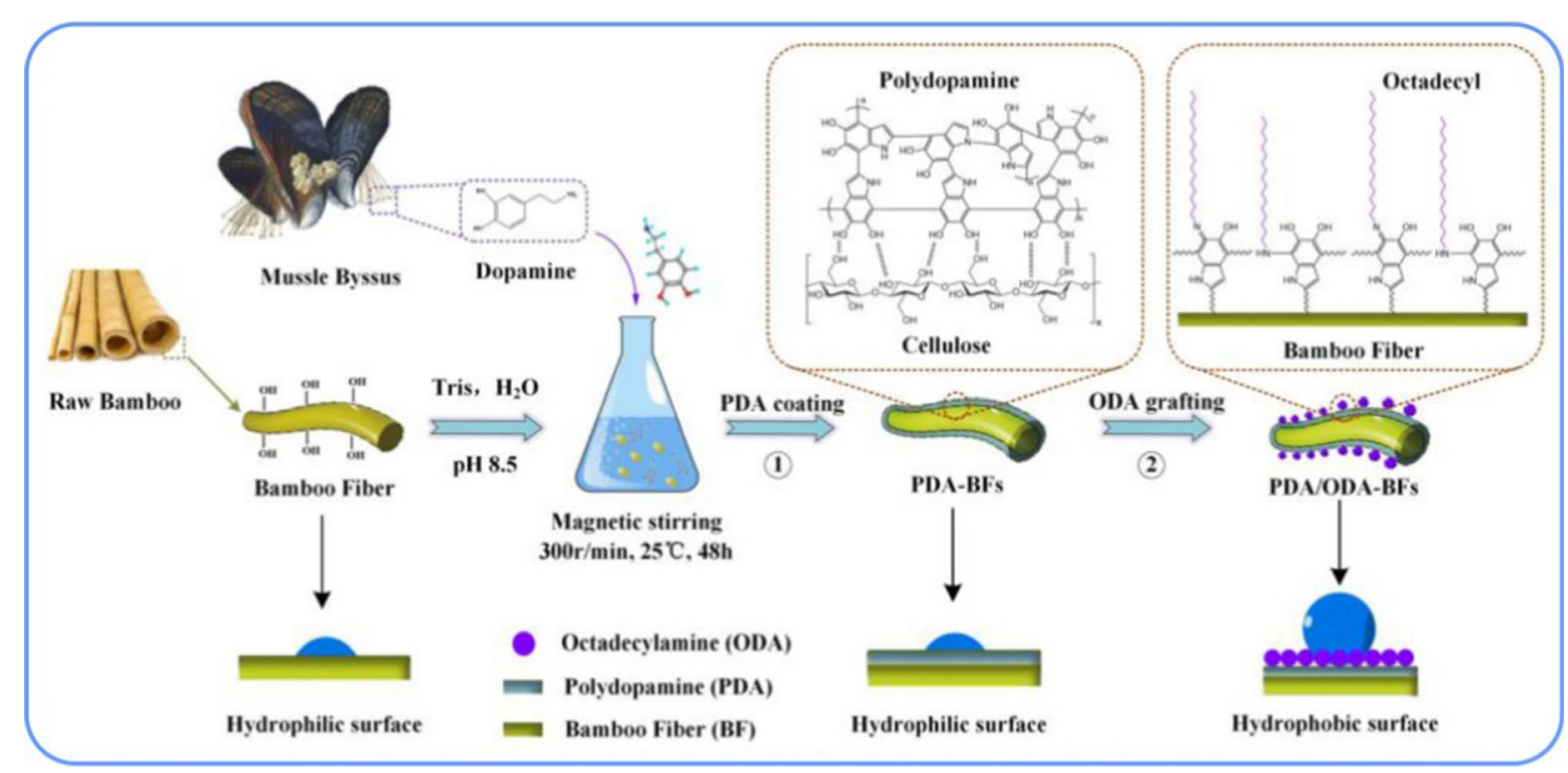

Fig. 11 The process of BFs modified by PDA coating and ODA grafting. Figure 11 reproduced with permission from Shuangbao Zhang. (Hong et al. 2018)

Publisher's Note Springer Nature remains neutral with regard to jurisdictional claims in published maps and institutional affiliations. 